\title{
Discrete Set Instance Identifier Data Type
}

National Cancer Institute

\section{Source}

National Cancer Institute. Discrete Set Instance Identifier Data Type. NCI Thesaurus.

Code C95647.

A data type comprised of a collection of instance identifier values that are distinct. 